\title{
Unexpected effects of NFKB in bone formation
}

It is well known that nuclear factor $\kappa \mathrm{B}$ (NFKB) has a crucial role in promoting osteoclast differentiation and bone resorption. Jia Chang and colleagues have now shown that this immune transcription factor also inhibits the function of mature bone-forming osteoblasts. Furthermore, specific inhibition of NFKB increases bone formation and protects against bone loss in an experimental mouse model of osteoporosis.

\section{$4 \mathrm{NF}_{\mathrm{K} B}$ inhibition enhances osteoblast function without affecting osteoclast function 77}

To study the effects of NFKB in mature osteoblasts, the osteoblast-specific bone $\gamma$-carboxyglutamate protein 2 (Bglap2) promoter was used to generate transgenic mice specifically expressing inhibitor of $\kappa \mathrm{B}$ kinase (IKK). Histomorphometric analysis at 2 weeks revealed increased trabecular bone mass and a higher rate of bone formation in Bglap2-IKK mice compared with their wild-type littermates. Furthermore, numbers of osteoblasts and osteoclasts were similar in both types of mice, as were serum levels of tartrateresistant acid phosphatase $5 \mathrm{~b}$, a marker of bone resorption and osteoclast function. These findings indicate that NFKB inhibition enhances osteoblast function without affecting osteoclast function. The observed increased in Fos-related antigen 1 expression in Bglap2-IKK mice could explain these positive effects.

The investigators then sought to determine whether bone formation could be maintained in ovariectomized Bglap2-IKK mice. Using micro-CT, they found only $12 \%$ loss of bone mineral density in the trabecular bone of Bglap2-IKK mice compared with $40 \%$ loss in wild-type animals. They also showed that proinflammatory cytokines, such as tumor necrosis factor and interleukin1, activate $\mathrm{NF \kappa B}$, leading to suppressed osteoblast function and inhibition of bone formation in ovariectomized mice.

The researchers hope that this approach could improve the future treatment of osteoporosis. "At present, many drugs used in the treatment of osteoporosis are bone resorption inhibitors, but they are unable to increase or restore bone mass," says lead investigator Cun-Yu Wang. "Therefore, identifying a drug that not only inhibits bone resorption but also promotes new bone formation would be a major therapeutic advance."

Negin Nassabeh

Original article Chang, J. et al. Inhibition of osteoblastic bone formation by nuclear factor-kB. Nat. Med. 15, 682-689 (2009). 